\title{
Correlated voltage probe model of relaxation in two Coulomb-coupled edge channels
}

\author{
Simon E. Nigg \\ Department of Physics, University of Basel, Klingelbergstrasse 82, 4056 Basel, Switzerland
}

\section{A R T I C L E I N F O}

Article history:

Received 12 May 2015

Received in revised form

18 August 2015

Accepted 3 September 2015

Available online 10 September 2015

Keywords:

Mesoscopic electron transport

Quantum Hall edge-states

Coulomb induced energy relaxation

Voltage probe model

Current noise

\begin{abstract}
A B S T R A C T
A phenomenological correlated voltage probe model is introduced to mimic the effects of inelastic scattering between particles in different conduction channels of a phase coherent conductor. As an illustration, the non-equilibrium distribution functions of two noisy co-propagating chiral edge channels of the integer quantum Hall effect are calculated and compared with recent experiments. The method is further applied to calculate the linear response current noise through an interacting Mach-Zehnder interferometer.
\end{abstract}

(c) 2015 Elsevier B.V. All rights reserved.

\section{Introduction}

Markus Büttiker was one of the pioneers of a very fruitful theoretical approach to mesoscopic electron transport, in which ample room is given to intuition. At the price of neglecting electron-electron interaction effects, ${ }^{1}$ this approach, usually referred to as the Landauer-Büttiker formalism, has demonstrated an impressive predictive power and is able to accurately describe a multitude of phenomena of electron transport through mesoscopic devices. Although developed in the 1980s it is still widely used by theoreticians and experimentalists alike all over the world. This may be largely attributed to its intuitive and nontechnical character.

Because electrons in a mesoscopic conductor carry electric charge, they interact with their electromagnetic environment. This interaction tends to suppress the effects of quantum coherence described by the elastic scattering approach. A microscopic description of this phenomenon - called decoherence - is often difficult to obtain as it requires a precise knowledge of the state and nature of the environment. Markus Büttiker extended the coherent scattering matrix approach to deal with inelastic processes in a phenomenological manner by introducing the concept of a fictitious voltage probe [1-3]. As its name suggests the latter is

\footnotetext{
E-mail address: simon.nigg@unibas.ch

${ }^{1}$ Neglecting electron-electron interaction in transport through gate defined 2DEG structures is often a better approximation than it may seem owing to screening and the emergence of weakly interacting quasi-particles.

a hypothetical (ideal) voltage probe attached to the conduction channel of the coherent conductor in such a way as to allow incoming particles to relax in energy before being injected back into the conductor. The strength of this approach lies in the fact that the entire system consisting of the conductor and the voltage probe can again be treated as a coherent conductor to which the elastic scattering matrix approach is applicable. Imposing certain constraints on the voltage probe yields an effective description of inelastic transport through the conductor. The degree of decoherence, in this phenomenological model, is controlled by the strength of the coupling between the voltage probe and the coherent conductor. It is worthwhile to note that the philosophy here is similar to that encountered in the open quantum systems approach to decoherence based on extending the Hilbert space of the system to include its environment [4]. The state of the full system is pure and obeys the Schrödinger equation and an effective description of the incoherent evolution is obtained by integrating out the degrees of freedom of the environment. The voltage probe model and the closely related dephasing probe model [5] have been widely used to make theoretical predictions [1-3,6-15] and analyze experimental data $[16,17]$.

Interaction effects between components of a mesoscopic conductor are notoriously difficult to describe within the scattering matrix formalism [18-21]. Even the traditional voltage probe model falls short here, because energy relaxation at two different probes is uncorrelated. In the present work, I would like to introduce a variant of the voltage probe model that allows one to describe phenomenologically the Coulomb interaction between 
two coherent mesoscopic conductors, i.e. a situation where the energy lost by one component is absorbed by the other. I call this the correlated voltage probe model (CVP). As we shall see, this model allows an effective description of interaction induced energy exchange between different channels of a mesoscopic conductor, within the non-interacting (single-particle) scattering matrix formalism. By its very nature, it does not provide a microscopic description of the (many-body) Coulomb interaction and no attempt will be made to derive it from first principles. Instead I will content myself with providing some comparison with other models and experimental data.

\section{Energy relaxation between two co-propagating edge channels}

To introduce the CVP model, let us consider the system schematically depicted in Fig. 1. It consists of two co-propagating edge states (ESs) in a typical gate-defined integer quantum Hall effect geometry patterned in a $2 \mathrm{D}$ electron gas (2DEG). The electron distribution of the outer ES is brought out of equilibrium at the leftmost quantum point contact (QPC). An electron passing this QPC in the outer (inner) ES gets reflected with probability $1-\mathcal{T}$ $(0)$ and transmitted with probability $\mathcal{T}(1)$. Hence the distribution functions of the inner and outer ESs right after the QPC $\left(x=0^{+}\right)$ are given by

$f_{i}^{(0)}(E) \equiv f_{i}\left(E, x=0^{+}\right)=\frac{1}{1+\exp \left(\beta\left(E-\mu_{2}\right)\right)}$

$$
\begin{aligned}
& f_{o}^{(0)}(E) \\
& \quad \equiv f_{o}\left(E, x=0^{+}\right)=\frac{\mathcal{T}}{1+\exp \left(\beta\left(E-\mu_{1}\right)\right)}+\frac{1-\mathcal{T}}{1+\exp \left(\beta\left(E-\mu_{2}\right)\right)},
\end{aligned}
$$

with the inverse temperature of the contacts $\beta=1 /\left(k_{\mathrm{B}} T\right)$. As the electrons propagate along the edge $(x>0)$, they may exchange energy via Coulomb interaction. This problem of energy relaxation along edge states of the integer quantum Hall effect is at the origin of a growing number of works [22-32]. A simple calculation shows that for energies close to the Fermi energy, the intra-ES interaction is negligible compared with the inter-ES interaction [23,25]. Hence we shall consider only the latter in what follows. Furthermore, it is expected and experimentally confirmed $[23,24]$ that for sufficient spatial separation between the two ESs, energy equilibration occurs on much shorter lengths than particle exchange. Hence we shall neglect inter-ES particle scattering.

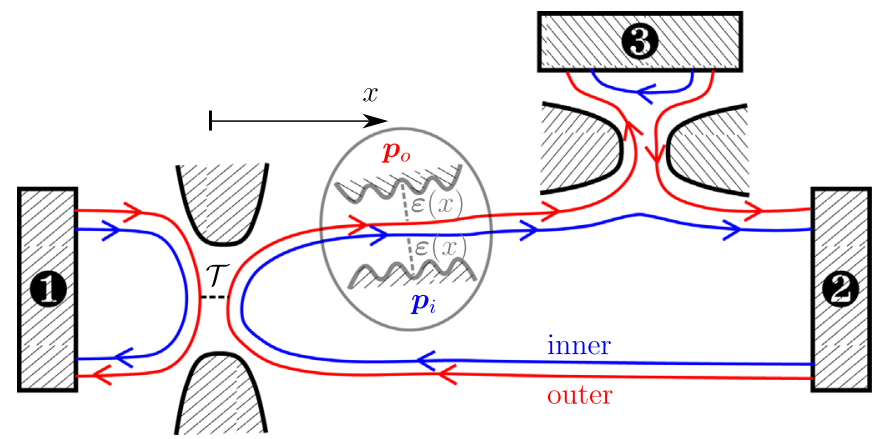

Fig. 1. Correlated voltage probe model for two out-of-equilibrium Coulomb-coupled edge channels.

\subsection{The correlated voltage probe model}

To simulate the effect of long range Coulomb interaction between the two ESs, we couple each ES to a fictitious voltage probe via a fictitious QPC. We shall consider the case where the distribution functions inside the voltage probes are given by equilibrium distributions $f_{p_{\sigma}}(E)=1 /\left[\exp \left\{\left(E-\mu_{p}^{\sigma}\right) /\left(k_{\mathrm{B}} T_{p}\right)\right\}+1\right]$ at the temperature $T_{p}$. Note that the latter may differ from the physical temperature $T$ of the real probes and, as we shall see later, is determined by the asymptotic (long distance) equilibrium state of the edge. Here $\sigma \in\{i, o\}$ labels the inner $(i)$ and outer (o) ES. As in the original voltage probe model [3], particle conservation in each ES is enforced by requiring that the current into the voltage probes vanishes at all times. This implies that both the average currents and the current fluctuations into the probes must vanish:

(i) Zero average current $\left\langle I_{p_{0}}\right\rangle=\left\langle I_{p_{i}}\right\rangle=0$.

(ii) Zero current fluctuations $\Delta I_{p_{o}}=\Delta I_{p_{i}}=0$.

The average total current into contact $\alpha$ is given by $\left\langle I_{\alpha}\right\rangle=(1 / e) \int d E \sum_{\beta} G_{\alpha \beta} \bar{f}_{\beta}(E)$, where $G_{\alpha \beta}=\left(e^{2} / h\right)\left[N_{\alpha} \delta_{\alpha \beta}-\operatorname{Tr}\left\{s_{\alpha \beta}^{\dagger} s_{\alpha \beta}\right\}\right]$ is the conductance from contact $\beta$ to contact $\alpha, N_{\alpha}$ is the number of open channels at contact $\alpha$ and $s_{\alpha \beta}$ the unitary elastic scattering matrix of the system including the voltage probes. We use the bar notation $\bar{f}_{\alpha}$ to denote the average distribution function in contact $\alpha$. Because we neglect inter-ES particle scattering, the scattering matrix is diagonal in the channel subspace, i.e.

$s_{\alpha \beta}=\left(\begin{array}{cc}s_{\alpha \beta}^{(i)} & 0 \\ 0 & s_{\alpha \beta}^{(o)}\end{array}\right)$.

The scattering matrices for the inner and outer channels are explicitly given by (we order the contacts as $\left(1,2,3, p_{0}, p_{i}\right)$ )

$$
\begin{aligned}
s^{(i)} & =\left(\begin{array}{ccccc}
1 & 0 & 0 & 0 & 0 \\
0 & i \sqrt{1-\varepsilon} & 0 & 0 & \sqrt{\varepsilon} \\
0 & 0 & 1 & 0 & 0 \\
0 & 0 & 0 & 1 & 0 \\
0 & \sqrt{\varepsilon} & 0 & 0 & i \sqrt{1-\varepsilon}
\end{array}\right), \\
S^{(0)} & =\left(\begin{array}{ccccc}
i \sqrt{1-\mathcal{T}} & \sqrt{\mathcal{T}} & 0 & 0 & 0 \\
0 & 0 & 1 & 0 & 0 \\
i \sqrt{\mathcal{T}} \sqrt{1-\varepsilon} & -\sqrt{1-\mathcal{T}} \sqrt{1-\varepsilon} & 0 & \sqrt{\varepsilon} & 0 \\
\sqrt{\mathcal{T}} \sqrt{\varepsilon} & i \sqrt{1-\mathcal{T}} \sqrt{\varepsilon} & 0 & i \sqrt{1-\varepsilon} & 0 \\
0 & 0 & 0 & 0 & 1
\end{array}\right)
\end{aligned}
$$

The resulting conductance matrix is therefore

$$
G=\frac{e^{2}}{h}\left(\begin{array}{ccccc}
T & -T & 0 & 0 & 0 \\
0 & 1+\varepsilon & -1 & 0 & -\varepsilon \\
-\mathcal{T}(1-\varepsilon) & -(1-\mathcal{T})(1-\varepsilon) & 1 & -\varepsilon & 0 \\
-T \varepsilon & -(1-\mathcal{T}) \varepsilon & 0 & \varepsilon & 0 \\
0 & -\varepsilon & 0 & 0 & \varepsilon
\end{array}\right) .
$$

Condition (i) fixes the average chemical potentials in the probes. Indeed $\left\langle I_{p_{i}}\right\rangle=(1 / e) \int d E \sum_{\beta} G_{p_{i} \beta} \bar{f}_{\beta}=0$, implies (for $\varepsilon \neq 0$ ) that $\int d E\left[\bar{f}_{p_{i}}-f_{2}\right]=0$, which, as shown in Appendix $A(a)$, even for Fermi distributions at different temperatures, implies $\bar{\mu}_{p_{i}}=\mu_{2}$. Similarly $\left\langle I_{p_{0}}\right\rangle=0$ implies $\bar{\mu}_{p_{o}}=\mathcal{T}_{\mu_{1}}+\mathcal{R} \mu_{2}$. Here $\mathcal{R}=1-\mathcal{T}$.

\subsection{Energy conservation and correlated probe temperature}

In order to simulate the energy exchange between the two ESs, we introduce the additional constraint of average energy conservation, which is most conveniently expressed as 
(iii) Zero average energy current $\left\langle j_{p_{0}}\right\rangle+\left\langle j_{p_{i}}\right\rangle=0$.

This condition correlates the two probes by fixing the probe temperature $T_{p}$ as follows. The average energy current into probe $\alpha$ is defined by $\left\langle j_{\alpha}\right\rangle=\frac{1}{\hbar} \int d E E \sum_{\beta} g_{\alpha \beta} \bar{f}_{\beta}$ where $g_{\alpha \beta}=\left(h / e^{2}\right) G_{\alpha \beta}$ is the dimensionless electrical conductance from $\beta$ to $\alpha$. The condition (iii) then leads to

$\int d E E\left[\bar{f}_{p_{i}}-f_{2}\right]+\int d E E\left[\bar{f}_{p_{o}}-\mathcal{R} f_{2}-\mathcal{T} f_{1}\right]=0$.

Using the integral computed in Appendix $A(b)$ : $\int d E E\left[f_{a}(E-\mu)-f_{b}(E)\right]=\left(\mu^{2} / 2\right)+\left(\pi^{2} / 6\right)\left(1 / a^{2}-1 / b^{2}\right)$ where $f_{\alpha}=$ $1 /[\exp (\alpha E)+1]$, one finds that Eq. (6) is equivalent to

$$
\frac{2 \pi^{2}}{3}\left[\left(k_{\mathrm{B}} T_{p}\right)^{2}-\left(k_{\mathrm{B}} T\right)^{2}\right]-\mathcal{R} \mathcal{T} \Delta \mu^{2}=0 \quad \Rightarrow \quad k_{\mathrm{B}} T_{p}=\sqrt{\left(k_{\mathrm{B}} T\right)^{2}+\frac{3}{2 \pi^{2}} \mathcal{R} \mathcal{T} \Delta \mu^{2}} .
$$

Here $\Delta \mu=\mu_{2}-\mu_{1}$. Expression (7) for the probe temperature coincides with the asymptotic temperature (for $x \rightarrow \infty$ ), predicted from energy equi-partitioning among the two ESs [23,25,26]. Note that conditions (i) and (iii) are sufficient to fully specify a CVP in thermal equilibrium. For an out-of-equilibrium probe model, additional conditions would in general be required.

\subsection{The distribution functions along the ESs}

The space dependence of the energy relaxation is simulated by making the transmission probability of the fictitious QPCs space dependent: $\varepsilon \rightarrow \varepsilon(x)$. The particular choice for the space-dependence is motivated by the following considerations. Physically, energy exchange between the electrons in the inner and outer ESs may take place anywhere between the QPC and a given point a distance $x$ away in the propagation direction. To simulate this, one may want to couple, with constant strength $\varepsilon_{0}, N$ equidistant correlated probes over a distance $x=N \Delta x$ along the edge as depicted in Fig. 2(a). The probability that an electron traveling a distance $x$ has visited at least one of the probes is given by $P_{N}=1-\left(1-\varepsilon_{0}\right)^{N}$. The idea is to replace the $N$ probes by a single probe with a space-dependent coupling strength $\varepsilon(x)$, which is given by the probability that an electron has been absorbed by at least one probe after a distance $x$ (see Fig. 2(b)). To this end we introduce the relaxation length $\ell_{\text {rel }}$ and define $\varepsilon_{0}=\Delta x / \ell_{\text {rel }}$ (supposing of course that $\Delta x \ll \ell_{\text {rel }}$ ). The probability that an electron has visited at least one probe after traveling a distance $x$ is now given

a
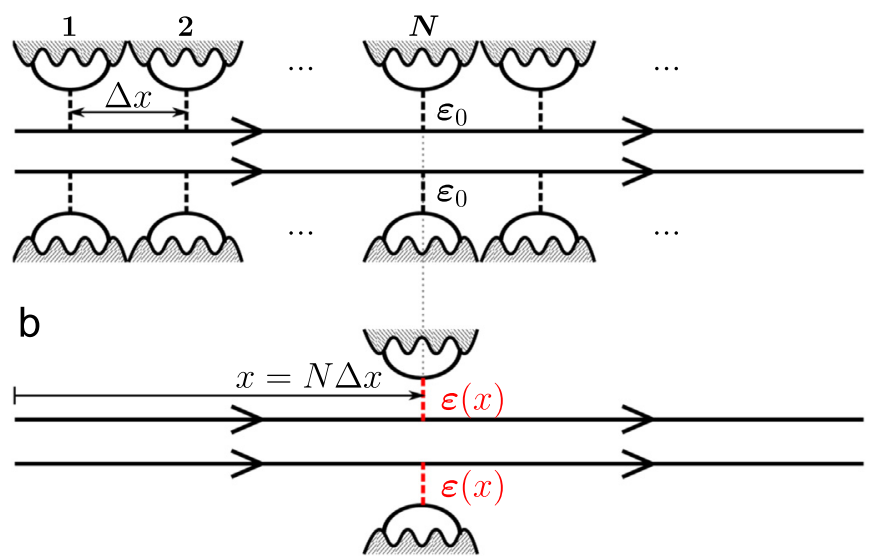

Fig. 2. (a) Multiple correlated voltage probes with constant coupling. (b) Equivalent single correlated voltage probe with space-dependent coupling. by

$\varepsilon(x)=1-\left(1-\frac{x}{N \ell_{\mathrm{rel}}}\right)^{N} \stackrel{N \rightarrow \infty, \Delta x \rightarrow 0}{\longrightarrow} 1-e^{-x / \ell_{\mathrm{rel}} .}$

With this definition of the probe transmission probability, the distribution functions in the inner and outer ESs a distance $x$ away from the leftmost QPC are simply given by

$f_{\alpha}(E, x)=[1-\varepsilon(x)] f_{\alpha}^{(0)}(E)+\varepsilon(x) f_{p_{\alpha}}(E)$

These expressions interpolate smoothly between the regimes of no equilibration $(\varepsilon=0)$ and full equilibration $(\varepsilon=1)$. Fig. 3 illustrates

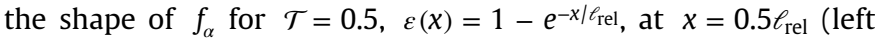
panel), $x=2 \ell_{\text {rel }}$ (center panel) and $x=10 \ell_{\text {rel }}$ (right panel).

\subsection{Excess temperature: comparison with experiments and a mi- croscopic theory}

We can use the CVP model to calculate the excess temperature of the quasi-particles $[23,24]$ along the ESs. The excess temperature is defined via the difference of heat currents between the actual distribution and a zero temperature equilibrium system with equal particle number:

$k_{\mathrm{B}} T_{\text {excess }, \alpha}=\sqrt{\frac{6}{\pi^{2}} \int d E\left[f_{\alpha}(E)-\theta\left(\tilde{\mu}_{\alpha}-E\right)\right]\left(E-\tilde{\mu}_{\alpha}\right)-\left(k_{\mathrm{B}} T\right)^{2}}$,

with $\tilde{\mu}_{o}=\mathcal{T} \mu_{1}+\mathcal{R} \mu_{2}$ and $\tilde{\mu}_{i}=\mu_{2}$. With the help of the integral evaluated in Appendix A(b), we find, for the outer ES, $k_{\mathrm{B}} T_{\text {excess }, o}=\sqrt{\varepsilon\left[\left(k_{\mathrm{B}} T_{p}\right)^{2}-\left(k_{\mathrm{B}} T\right)^{2}\right]+\left(3 / \pi^{2}\right)(1-\varepsilon) \Delta \mu^{2} \mathcal{R T}}$, which with $T_{p}$ given by Eq. (7) above reduces to

$k_{\mathrm{B}} T_{\text {excess }, o}=|\Delta \mu| \sqrt{\frac{3}{\pi^{2}} \mathcal{R T}\left(1-\frac{\varepsilon}{2}\right)}$.

Thus assuming $\varepsilon$ does not depend on voltage or temperature, the correlated VP model predicts an excess temperature that is linear in the voltage bias and temperature independent. This is to be contrasted with the (weak) nonlinearity and temperature dependence found in $[25,26]$. In Fig. 4 we show the comparison between Eq. (11) with $\varepsilon(x)=1-e^{-x / \theta_{\text {rel }}}$ and the experimental data of Altimiras et al. [23], for a relaxation length $\ell_{\text {rel }}=2 \mu \mathrm{m}$. On the same figure we also show the best-fit result of the microscopic theory [25] with two channels (dashed curves).

\subsection{Current fluctuation spectra}

We next apply the CVP model to compute the zero-frequency current noise of the ESs. Following [33], let us write the current into contact $\alpha$ as $I_{\alpha}=(1 / e) \int d E \sum_{\beta} G_{\alpha \beta} f_{\beta}+\delta I_{\alpha}=\left\langle I_{\alpha}\right\rangle+\Delta I_{\alpha}$, where $\delta I_{\alpha}$ denotes the intrinsic current fluctuations in contact $\alpha$, while $\Delta I_{\alpha}$ also includes the contribution from the fluctuating distribution. We see that condition (ii) implies that $\delta I_{p_{i}}=-\varepsilon(e / h)\left(\mu_{p_{i}}-\mu_{2}\right)=-\varepsilon(e / h) \Delta \mu_{p_{i}}$ and $\delta I_{p_{o}}=-\varepsilon(e / h)\left(\mu_{p_{o}}-\mathcal{T}_{\mu_{1}}-\mathcal{R} \mu_{2}\right)=-\varepsilon(e / h) \Delta \mu_{p_{o}}$. This in turn implies that $\Delta I_{\alpha}=\delta I_{\alpha}+(1 / e) \sum_{p=p_{i}, p_{o}} G_{\alpha p} \Delta \mu_{p}$. In particular we thus have $\Delta I_{2}=\delta I_{2}+\delta I_{p_{i}}$ and $\Delta I_{3}=\delta I_{3}+\delta I_{p_{0}}$. Since, because of chirality (no electrons flow from the voltage probes to contact 1 ), $\Delta I_{1}=\delta I_{1}$, we have $\sum_{\alpha} \Delta I_{\alpha}=\sum_{\alpha} \delta I_{\alpha}=0$, where the last equality follows from current conservation.

\subsubsection{Auto-correlations}

With the results from the previous section, we see that the auto-correlation fluctuation spectrum of the inner ES measured at 

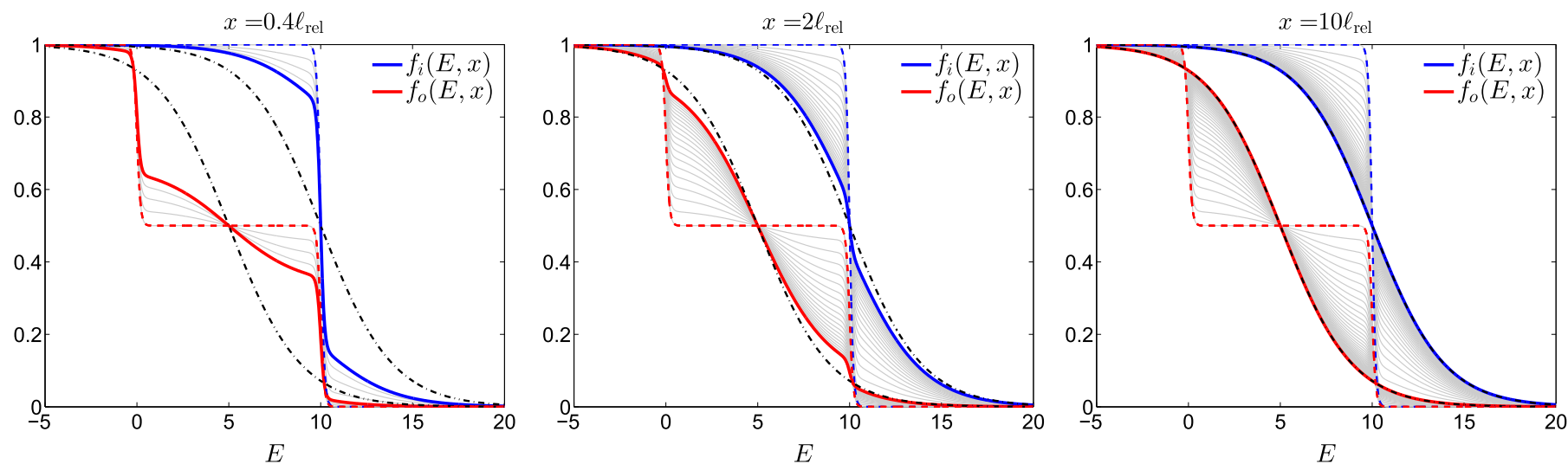

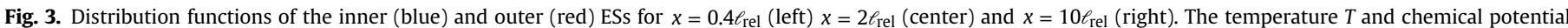

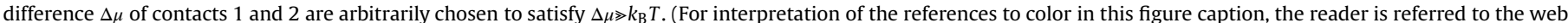
version of this paper.)

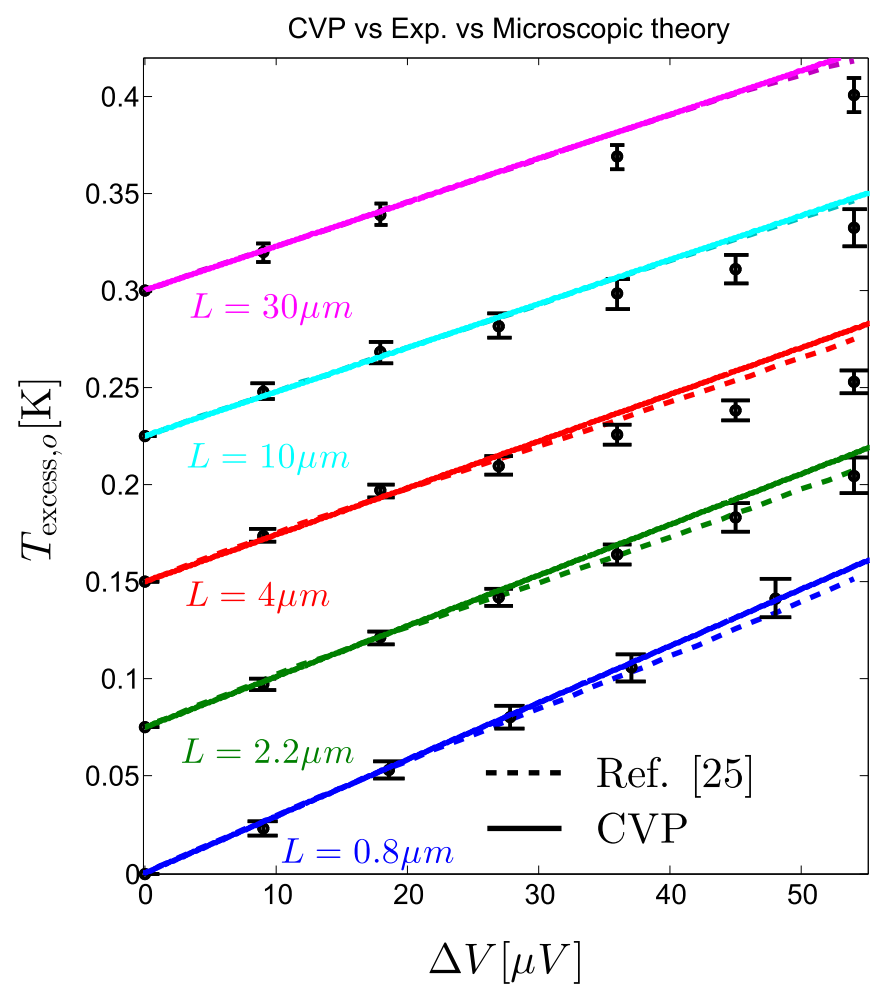

Fig. 4. Comparison of the CVP model with experimental data [23] and previous microscopic theory [25]. Shown is the excess temperature of the outer ES as a function of $\Delta \mu=e \Delta V$ for different propagation lengths. Curves for different lengths are shifted upward by increments of $75 \mathrm{mK}$ for clarity.

contact 2 is given by $S_{22}=\left\langle\Delta I_{2} \Delta I_{2}\right\rangle=S_{22}^{0}+S_{p_{i} p_{i}}^{0}+S_{p_{i} 2}^{0}+S_{2 p_{i}}^{0}$, where $S_{\alpha \beta}^{0}=\left\langle\delta I_{\alpha} \delta I_{\beta}\right\rangle$ is the bare fluctuation spectrum of the system with only elastic scattering given by $^{2}$

$S_{\alpha \beta}^{0}=\frac{2 e^{2}}{h} \int d E \sum_{\gamma, \lambda} \operatorname{Tr}\left\{A_{\gamma \lambda}^{\alpha} A_{\lambda \gamma}^{\beta}\right\} f_{\gamma}\left(1-f_{\lambda}\right)$,

with $A_{\gamma \lambda}^{\alpha}=\delta_{\alpha \gamma} \delta_{\alpha \lambda} \mathbf{1}_{\alpha}-s_{\alpha \gamma}^{\dagger} s_{\alpha \lambda}$. Explicitly we find

\footnotetext{
${ }^{2}$ N.B. we consider here the symmetrized noise.
}

$$
\begin{aligned}
S_{22}^{0} & =\frac{2 e^{2}}{h} \int d E\left\{\varepsilon^{2} f_{p_{i}}\left(1-f_{p_{i}}\right)+f_{2}\left(1-f_{2}\right)+(1-\varepsilon)^{2} f_{1}\left(1-f_{1}\right)+\varepsilon(1-\varepsilon)\left[f_{1}\left(1-f_{p_{i}}\right)+f_{p_{i}}\left(1-f_{1}\right)\right]\right\} \\
& =\frac{2 e^{2}}{h}\left\{\varepsilon^{2} k_{\mathrm{B}} T_{p}+k_{\mathrm{B}} T(1-\varepsilon)^{2}+k_{\mathrm{B}} T+\varepsilon(1-\varepsilon)\left[f_{1}\left(1-f_{p_{i}}\right)+f_{p_{i}}\left(1-f_{1}\right)\right]\right\}, \\
S_{p_{i}}^{0} p_{i} & =\frac{2 e^{2}}{h} \int d E\left\{\varepsilon^{2} f_{p_{i}}\left(1-f_{p_{i}}\right)+\varepsilon^{2} f_{f_{1}}\left(1-f_{1}\right)+\varepsilon(1-\varepsilon)\left[f_{1}\left(1-f_{p_{i}}\right)+f_{p_{i}}\left(1-f_{1}\right)\right]\right\} \\
& =\frac{2 e^{2}}{h}\left\{\varepsilon^{2} k_{\mathrm{B}}\left(T_{p}+T\right)+\varepsilon(1-\varepsilon)\left[f_{1}\left(1-f_{p_{i}}\right)+f_{p_{i}}\left(1-f_{1}\right)\right]\right\}, \\
S_{p_{i} 2}^{0}= & S_{2 p_{i}}^{0}=-\frac{2 e^{2}}{h} \int d E\left\{\varepsilon^{2} f_{p_{i}}\left(1-f_{p_{i}}\right)-\varepsilon(1-\varepsilon)\left[f_{1}\left(1-f_{1}\right)+f_{1}\left(1-f_{p_{i}}\right)+f_{p_{i}}\left(1-f_{1}\right)\right]\right\} \\
& =\frac{2 e^{2}}{h}\left\{\varepsilon(1-\varepsilon)\left[f_{p_{i}}\left(1-f_{1}\right)+f_{1}\left(1-f_{p_{i}}\right)\right]+\varepsilon(1-\varepsilon) k \mathrm{~B} T-\varepsilon^{2} k_{\mathrm{B}} T_{p}\right\} .
\end{aligned}
$$

Summing all terms we obtain

$S_{22}=\left(4 e^{2} / h\right) k_{\mathrm{B}} T=S_{11}^{i}$.

Thus we find that the noise spectrum in contact 2 is the same as in the inner ES in contact 1, independently of the probe temperature $T_{p}$.

Similarly we have $S_{33}=\left\langle\Delta I_{3} \Delta I_{3}\right\rangle=S_{33}^{0}+S_{p_{0} p_{0}}^{0}+S_{p_{0} 3}^{0}+S_{3 p_{0}}^{0}$ and we find, following similar steps:

$S_{33}=\left(2 e^{2} / h\right)\left\{2 k_{\mathrm{B}} T(1-\mathcal{R T})+\mathcal{R T} \Delta \mu \operatorname{coth}(\beta \Delta \mu / 2)\right\}$,

which is nothing else but the thermal noise plus the noise generated at the QPC in the outer ES, in the absence of the probe.

\subsubsection{Cross-correlations}

For the current cross-correlation $S_{23}=\left\langle\Delta I_{2} \Delta I_{3}\right\rangle=\left\langle\delta I_{2} \delta I_{3}\right\rangle+$ $\left\langle\delta I_{2} \delta I_{p_{0}}\right\rangle+\left\langle\delta I_{p_{i}} \delta I_{3}\right\rangle+\left\langle\delta I_{p_{i}} \delta I_{p_{0}}\right\rangle$, we find $S_{23}=S_{32}=\left(2 e^{2} / h\right) \mathcal{T} k_{\mathrm{B}} T=$ $(\mathcal{T} / 2) S_{11}$, which again is independent of the probes. According to this result, only electrons coming from the same contact, the proportion of which is $\mathcal{T}$, contribute to the (positive) cross-correlations between the two channels, which vanish at zero temperature.

Therefore, the correlated voltage probe model predicts that energy exchange between the ESs does not affect the zero frequency noise (auto- and cross-correlation) along the ESs.

\subsection{Discussion}

The strength of the correlated voltage probe model lies in the simple analytic predictions it makes for the energy relaxation of electrons along two co-propagating ESs. In particular, assuming a bias-independent relaxation length $\ell_{\text {rel }}$, the excess temperature is predicted to scale linearly with bias voltage. For $\ell_{\text {rel }}=20 \mu \mathrm{m}$, consistent with recent experiments [24], we find reasonable qualitative agreement between the correlated voltage probe model and experiments. 


\section{Application to the Mach-Zehnder interferometer}

We next apply the CVP model to the electronic Mach-Zehnder interferometer at filling factor $\nu=2$ [14,34-46]. This system is schematically depicted in Fig. 5 . We attach a voltage probe to each arm via fictitious QPCs with transmission probability $\varepsilon$. We are interested primarily in the average current and the current noise into probe 3 .

\subsection{Gauge invariance and interaction}

Before discussing the predictions of the correlated voltage probe for the interacting Mach-Zehnder interferometer, we briefly address an important problem, which arises in the non-interacting theory of non-linear transport, as often emphasized by Markus Büttiker and his co-workers $[47,48]$. In the linear transport regime, transport properties are determined by the equilibrium system (i.e. with zero bias voltage applied). In this situation the energy dependence of the scattering matrix can be entirely attributed to the Aharonov-Bohm phase acquired by the electron wave function exploring the different arms of the interferometer. The current for example is then simply a sum of convolutions of conductances and equilibrium distribution functions. Importantly this quantity and all other transport quantities are gauge invariant, i.e. invariant under a global shift of the potentials in the reservoirs. Although gauge invariance follows from very general principles and should therefore always be valid, it is easy to see that a non-interacting theory in the nonlinear transport regime, where the bias dependence enters solely via the distribution functions but not in the scattering matrix, is not gauge invariant in general. One can avoid this problem in special situations in which the scattering matrix elements can be taken to be energy independent. However the current through the Mach-Zehnder is sensitive to the phase, which depends on energy and we thus face the problem that the non-interacting approximation is unphysical in the nonlinear transport regime [49]. In the following we therefore consider the linear response regime, where the non-interacting limit is gauge invariant.

\subsection{Average current through the interferometer}

The average current into probe 3 is given by $\left\langle I_{3}\right\rangle=(1 / e) \int d E \sum_{\beta} G_{3 \beta} \bar{f}_{\beta}$, where the index $\beta$ runs over all contacts including the probes and $G_{\alpha \beta}=\left(e^{2} / h\right)\left[N_{\alpha} \delta_{\alpha \beta}-\operatorname{Tr}\left\{s_{\alpha \beta}^{\dagger} s_{\alpha \beta}\right\}\right]$. Since we neglect inter-ES scattering, the scattering matrix is diagonal in the channel subspace as previously. We have

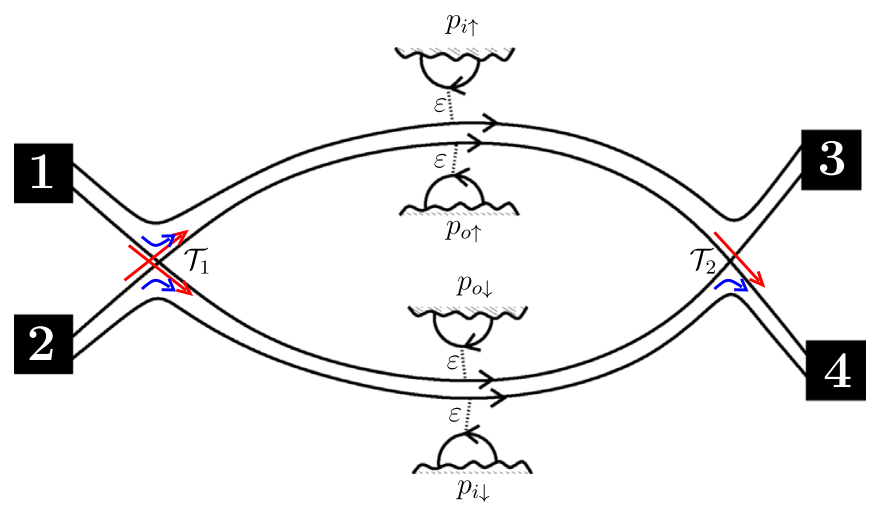

Fig. 5. Schematics of the electronic Mach-Zehnder interferometer. The upper (lower) arm of the interferometer is labeled with $\uparrow(\downarrow)$.

$$
\begin{aligned}
& S^{(i)}=\left(\begin{array}{cccc}
s_{p_{i \uparrow} 1}^{(i)} & 0 & s_{p_{i \uparrow} p_{i \uparrow}}^{(i)} & 0 \\
0 & s_{p_{i \downarrow} 2}^{(i)} & 0 & s_{p_{i \downarrow} p_{i \downarrow}}^{(i)} \\
S_{31}^{(i)} & 0 & s_{3 p_{i \uparrow}}^{(i)} & 0 \\
0 & s_{42}^{(i)} & 0 & s_{4 p_{i \downarrow}}^{(i)}
\end{array}\right), \\
& S^{(o)}=\left(\begin{array}{cccc}
s_{p_{o \uparrow 1}}^{(o)} & s_{p_{0 \uparrow} 2}^{(o)} & s_{p_{o \uparrow} p_{0 \uparrow}}^{(o)} & 0 \\
s_{p_{o \downarrow} 1}^{(o)} & s_{p_{o \downarrow} 2}^{(o)} & 0 & s_{p_{o \downarrow} p_{o \downarrow}}^{(o)} \\
s_{31}^{(o)} & s_{32}^{(o)} & s_{3 p_{o \uparrow}}^{(o)} & s_{3 p_{o \downarrow}}^{(o)} \\
s_{41}^{(o)} & s_{42}^{(o)} & s_{4 p_{o \uparrow}}^{(o)} & s_{4 p_{o \downarrow}}^{(o)}
\end{array}\right)
\end{aligned}
$$

with the elements

$$
\begin{aligned}
& s_{p_{i \uparrow} 1}^{(i)}=s_{p_{i \downarrow} 2}^{(i)}=s_{3 p_{i \uparrow}}^{(i)}=s_{4 p_{i \downarrow}}^{(i)}=\sqrt{\varepsilon} \\
& s_{p_{i \uparrow} p_{i \uparrow}}^{(i)}=s_{p_{i \downarrow}}^{(i)} p_{i \downarrow}=s_{31}^{(i)}=s_{42}^{(i)}=i \sqrt{1-\varepsilon} \\
& s_{p_{0 \uparrow} 1}^{(o)}=s_{p_{0 \downarrow}{ }^{2}}^{(o)}=\sqrt{\mathcal{T}_{1} \varepsilon}, \quad s_{p_{0 \uparrow^{2}}}^{(o)}=i \sqrt{1-\mathcal{T}_{1}} \sqrt{\varepsilon}, \quad s_{p_{0 \uparrow} p_{0 \uparrow}}^{(o)}=i \sqrt{1-\varepsilon}, \quad s_{p_{0 \downarrow} 1}^{(o)}=i \sqrt{1-\mathcal{T}_{1}} \sqrt{\varepsilon} \\
& s_{p_{0 \downarrow} p_{0 \downarrow}}^{(o)}=i \sqrt{1-\varepsilon}, \quad s_{31}^{(o)}=i \sqrt{\mathcal{T}_{1} \mathcal{T}_{2}} \sqrt{1-\varepsilon}-i e^{i \phi} \sqrt{1-\mathcal{T}_{1}} \sqrt{1-\mathcal{T}_{2}} \sqrt{1-\varepsilon}, \quad s_{3 p_{0 \uparrow}}^{(o)}=\sqrt{\mathcal{T}_{2} \varepsilon} \\
& s_{32}^{(o)}=-\sqrt{1-\mathcal{T}_{1}} \sqrt{\mathcal{T}_{2}} \sqrt{1-\varepsilon}-e^{i \phi} \sqrt{\mathcal{T}_{1}} \sqrt{1-\mathcal{T}_{2}} \sqrt{1-\varepsilon}, \quad s_{3 p_{0 \downarrow}}^{(o)}=i e^{i \phi_{\sqrt{\varepsilon}} \sqrt{1-\mathcal{T}_{2}}} \\
& s_{41}^{(o)}=-\sqrt{\mathcal{T}_{1}} \sqrt{1-\mathcal{T}_{2}} \sqrt{1-\varepsilon}-e^{i \phi} \sqrt{1-\mathcal{T}_{1}} \sqrt{\mathcal{T}_{2}} \sqrt{1-\varepsilon}, \quad s_{4 p_{0 \downarrow}}^{(o)}=e^{i \phi} \sqrt{\varepsilon} \sqrt{\mathcal{T}_{2}} \\
& s_{42}^{(o)}=i e^{i \phi} \sqrt{\mathcal{T}_{1}} \sqrt{1-\varepsilon} \sqrt{\mathcal{T}_{2}}-i \sqrt{1-\mathcal{T}_{1}} \sqrt{1-\mathcal{T}_{2}} \sqrt{1-\varepsilon}, \quad s_{4 p_{0 \uparrow}}^{(o)}=i \sqrt{\varepsilon} \sqrt{1-\mathcal{T}_{2}}
\end{aligned}
$$

Similarly the conductance can be split into a sum of conductances for each channel: $G=G^{(i)}+G^{(o)}$. The conductance matrices per channel are given by (in units of $e^{2} / h$ )

$$
\begin{aligned}
G^{(i)}= & \frac{e^{2}}{h}\left(\begin{array}{cccc}
-\varepsilon & 0 & \varepsilon & 0 \\
0 & -\varepsilon & 0 & \varepsilon \\
\varepsilon-1 & 0 & -\varepsilon & 0 \\
0 & \varepsilon-1 & 0 & -\varepsilon
\end{array}\right), \\
G^{(o)} & =\left(\begin{array}{cccc}
-\mathcal{T}_{1} \varepsilon & \left(\mathcal{T}_{1}-1\right) \varepsilon & \varepsilon & 0 \\
\left(\mathcal{T}_{1}-1\right) \varepsilon & -\mathcal{T}_{1} \varepsilon & 0 & \varepsilon \\
A(\phi) & B(\phi) & -\varepsilon \mathcal{T}_{2} & \varepsilon\left(\mathcal{T}_{2}-1\right) \\
B(\varepsilon, \phi) & A(\varepsilon, \phi) & \varepsilon\left(\mathcal{T}_{2}-1\right) & -\varepsilon \mathcal{T}_{2}
\end{array}\right)
\end{aligned}
$$

with

$A(\varepsilon, \phi)=(\varepsilon-1)\left[\mathcal{T}_{1} \mathcal{T}_{2}+\mathcal{R}_{1} \mathcal{R}_{2}-2 \sqrt{T_{1} \mathcal{T}_{2} \mathcal{R}_{1} \mathcal{R}_{2}} \cos (\phi)\right]$

$B(\varepsilon, \phi)=(\varepsilon-1)\left[T_{1} \mathcal{R}_{2}+\mathcal{R}_{1} \mathcal{T}_{2}+2 \sqrt{T_{1} \mathcal{T}_{2} \mathcal{R}_{1} \mathcal{R}_{2}} \cos (\phi)\right]$.

Note that $A+B=\varepsilon-1$. The currents per channel into probe 3 are thus

$$
\begin{aligned}
\left\langle I_{3}^{(i)}\right\rangle & =\frac{e}{h} \int d E\left[(1-\varepsilon) f_{1}+\varepsilon f_{p_{i \uparrow}}-f_{3}\right] \\
& =\frac{e}{h}\left[(1-\varepsilon)\left(\mu_{1}-\mu_{3}\right)+\varepsilon\left(\mu_{p_{i \uparrow}}-\mu_{3}\right)\right], \\
\left\langle I_{3}^{(o)}\right\rangle & =\frac{e}{h} \int d E\left[\varepsilon \mathcal{T}_{2} f_{p_{o \uparrow}}+\varepsilon \mathcal{R}_{2} f_{p_{o \downarrow}}-A(\varepsilon, \phi) f_{1}-B(\varepsilon, \phi) f_{2}-f_{3}\right] .
\end{aligned}
$$

Following the same steps as in Section 2.1, it is easily shown that the chemical potentials of the probes are given by

$\mu_{p_{i \uparrow}}=\mu_{1}, \quad \mu_{p_{i \downarrow}}=\mu_{2}, \quad \mu_{p_{o \uparrow}}=\mathcal{T}_{1} \mu_{1}+\mathcal{R}_{1} \mu_{2}, \quad \mu_{p_{o \downarrow}}=\mathcal{R}_{1} \mu_{1}+\mathcal{T}_{1} \mu_{2}$.

This follows again from the constraint of zero average current into each probe. Therefore we find $\left\langle I_{3}^{(i)}\right\rangle=(e / h)\left[\mu_{1}-\mu_{3}\right]$ and

$$
\begin{aligned}
\left\langle I_{3}^{(o)}\right\rangle= & \frac{e}{h}\left[\left(\mathcal{T}_{1} \mathcal{T}_{2}+\mathcal{R}_{1} \mathcal{R}_{2}\right)\left[\mu_{1}-\mu_{3}\right]\right. \\
& \left.+\left(\mathcal{R}_{1} \mathcal{T}_{2}+\mathcal{T}_{1} \mathcal{R}_{2}\right)\left[\mu_{2}-\mu_{3}\right]-2(1-\varepsilon) \sqrt{\mathcal{T}_{1} \mathcal{T}_{2} \mathcal{R}_{1} \mathcal{R}_{2}} \int d E \cos (\phi)\left[f_{1}-f_{2}\right]\right]
\end{aligned}
$$

The average current in the inner channel is unaffected by the probes, as expected, since current is conserved along this channel, but the current in the outer channel is affected by the probes. This 
is expressed in (21) by the suppression of the interference term $2(1-\varepsilon) \sqrt{\mathcal{T}_{1} \mathcal{T}_{2} \mathcal{R}_{1} \mathcal{R}_{2}} \int d E \cos (\phi)\left(f_{1}-f_{2}\right)$. Thus we see that the decohering effect of the probe and the phase averaging effect of the energy dependence of the phase factorize [14,34]. Nothing here depends on the temperature of the probes. The same results are obtained with uncorrelated probes.

We note that the result of Eq. (21) is not gauge invariant as it stands. However in the linear response regime we have $f_{1}-f_{2}=\Delta \mu f^{\prime}(E)$ with $\Delta \mu=\mu_{2}-\mu_{1}$ and so the current becomes

$\left\langle I_{3}^{(o)}\right\rangle=\frac{e}{h}\left[\left(\mathcal{T}_{1} \mathcal{T}_{2}+\mathcal{R}_{1} \mathcal{R}_{2}\right)\left[\mu_{1}-\mu_{3}\right]\right.$

$$
\left.+\left(\mathcal{R}_{1} \mathcal{T}_{2}+\mathcal{T}_{1} \mathcal{R}_{2}\right)\left[\mu_{2}-\mu_{3}\right]-2 \Delta \mu(1-\varepsilon) \sqrt{\mathcal{T}_{1} \mathcal{T}_{2} \mathcal{R}_{1} \mathcal{R}_{2}} \int d E \cos (\phi) f^{\prime}(E)\right] .
$$

which is gauge invariant. The relative phase $\phi$ is given by the sum of the Aharonov-Bohm phase $\Delta \phi_{A B}=\Phi / \Phi_{0}$, where $\Phi / \Phi_{0}$ is the external magnetic flux through the loop of the interferometer divided by the flux quantum, and the energy dependent path-length phase difference which we take to be $\Delta \phi=E\left(L_{\uparrow}-L_{\downarrow}\right) /\left(\hbar v_{D}\right)$. For simplicity we have assumed equal velocities in both ESs and a linear dispersion relation. One can evaluate the energy integral as shown in Appendix $A(c)$ and finally obtain

$\left\langle I_{3}^{(o)}\right\rangle=\frac{e}{h}\left[\left(\mathcal{T}_{1} \mathcal{T}_{2}+\mathcal{R}_{1} \mathcal{R}_{2}\right)\left[\mu_{1}-\mu_{3}\right]\right.$

$$
\left.+\left(\mathcal{R}_{1} \mathcal{T}_{2}+\mathcal{T}_{1} \mathcal{R}_{2}\right)\left[\mu_{2}-\mu_{3}\right]+2 \Delta \mu(1-\varepsilon) \sqrt{\mathcal{T}_{1} \mathcal{T}_{2} \mathcal{R}_{1} \mathcal{R}_{2}} \frac{\pi k_{\mathrm{B}} T \Delta L /\left(\hbar v_{D}\right)}{\sinh \left[\pi k_{\mathrm{B}} T \Delta L /\left(\hbar v_{D}\right)\right]} \cos \left(\Delta \phi_{A B}\right)\right],
$$

with $\Delta L=\left|L_{\uparrow}-L_{\downarrow}\right|$. Thus the energy dependence of the phase leads, at finite temperature and for different arm lengths, to an additional ( $\varepsilon$ independent) suppression of the oscillation amplitude by a factor $\pi k_{\mathrm{B}} T \Delta L /\left(\hbar v_{D}\right) / \sinh \left[\pi k_{\mathrm{B}} T \Delta L /\left(\hbar v_{D}\right)\right]$. This contribution to decoherence is usually called phase averaging. In the experimentally interesting regime one may ${ }^{3}$ have $k_{\mathrm{B}} T \ll \hbar v_{D} / \Delta L$. In this case the suppression factor is well approximated by $\left.1-)\left(\pi k_{\mathrm{B}} T\right)^{2} / 6\right)\left(\Delta L / \hbar v_{D}\right)^{2}$. In the opposite regime of high temperature $k_{\mathrm{B}} T \gg \hbar v_{D} / \Delta L$ the interference term is exponentially suppressed $\sim \exp \left[-\pi k_{\mathrm{B}} T \Delta L /\left(\hbar v_{D}\right)\right]$. An exponential suppression at high temperatures and a $T^{2}$ behavior of interference visibility at low temperatures has been observed in $[43,50]$. Note that an exponential suppression with temperature could also arise in the absence of thermal smearing if $\ell_{\text {rel }} \sim 1 / T$ [43].

\subsection{Current noise through the interferometer}

We consider the case where energy is conserved separately in each arm of the interferometer, which is physically reasonable when the two arms are well isolated from each other and do not interact. Thus we impose that the total energy current into the probes of each arm vanishes. Doing so we obtain, as before, for the temperatures of the probes

$k_{\mathrm{B}} T_{p_{\uparrow}}=k_{\mathrm{B}} T_{p \downarrow}=\sqrt{\left(k_{\mathrm{B}} T\right)^{2}+\frac{3}{2 \pi^{2}} \mathcal{R}_{1} \mathcal{T}_{1}\left(\mu_{1}-\mu_{2}\right)^{2}} \equiv k_{\mathrm{B}} T_{p}$.

Notice that the temperatures of the probes in both arms are the same and independent of the phase.

Similarly as in Section 2.1, conservation of current fluctuations into and out of the probes yields the relations $\Delta I_{3}=\delta I_{3}+\delta I_{p_{i \uparrow}}+\mathcal{T}_{2} \delta I_{p_{0 \uparrow}}+\mathcal{R}_{2} \delta I_{p_{o \downarrow}}$ and $\quad \Delta I_{4}=\delta I_{4}+\delta I_{p_{i \downarrow}}+\mathcal{R}_{2} \delta I_{p_{0 \uparrow}}+$ $\mathcal{T}_{2} \delta I_{p_{0 \downarrow}}$. Since $\Delta I_{1}=\delta I_{1}$ and $\Delta I_{2}=\delta I_{2}$, we see that $\sum_{i=1}^{4} \Delta I_{i}=\sum_{i} \delta I_{i}=0$. In order to simplify the calculation somewhat, we split up the current fluctuations into the channel contributions. Thus $\Delta I_{3}=\Delta I_{3}^{(i)}+\Delta I_{3}^{(o)}$ with $\Delta I_{3}^{(i)}=\delta I_{3}^{(i)}+\delta I_{p_{i \uparrow}}$ and

\footnotetext{
${ }^{3}$ For example for $\Delta L=1 \mu \mathrm{m}, v_{D}=10^{5} \mathrm{~m} / \mathrm{s}$ one has $\hbar v_{D} / \Delta L=764 \mathrm{mK}$, while the
} electronic temperatures can be as low as $T \approx 20 \mathrm{mK}$.
$\Delta I_{3}^{(o)}=\delta I_{3}^{(o)}+\mathcal{T}_{2} \delta I_{p_{0 \uparrow}}+\mathcal{R}_{2} \delta I_{p_{o \downarrow}}$. Then $\quad\left\langle\Delta I_{3} \Delta I_{3}\right\rangle=\left\langle\Delta I_{3}^{(i)} \Delta I_{3}^{(i)}\right\rangle+$ $\left\langle\Delta I_{3}^{(o)} \Delta I_{3}^{(o)}\right\rangle+\left\langle\Delta I_{3}^{(i)} \Delta I_{3}^{(o)}\right\rangle+\left\langle\Delta I_{3}^{(o)} \Delta I_{3}^{(i)}\right\rangle$. Since current is conserved separately in each ES, we have $\Delta I_{3}^{(i)}=\delta I_{3}^{(i)}+\delta I_{p_{i \uparrow}}^{(i)}=-\delta I_{1}^{i}$ so that $\left\langle\Delta I_{3}^{(i)} \Delta I_{3}^{(i)}\right\rangle=S_{11}^{(i)}=\left(4 e^{2} / h\right) k_{\mathrm{B}} T$. Hence the noise in the inner ES is, as expected, unaffected by the probes. Explicitly we further find, after some lengthy algebra that

$\left\langle\Delta I_{3}^{(i)} \Delta I_{3}^{(o)}\right\rangle=\left\langle\Delta I_{3}^{(o)} \Delta I_{3}^{(i)}\right\rangle=\frac{2 e^{2}}{h} \int d E j(E) f_{1}\left(1-f_{1}\right)$,

where we have introduced the density

$j(E) \equiv \mathcal{T}_{1} \mathcal{T}_{2}+\mathcal{R}_{1} \mathcal{R}_{2}-2(1-\varepsilon) \sqrt{\mathcal{T}_{1} \mathcal{T}_{2} \mathcal{R}_{1} \mathcal{R}_{2}} \cos (\phi)$.

In Appendix $A(c)$ we evaluate the integral over energy and find

$\left\langle\Delta I_{3}^{(o)} \Delta I_{3}^{(i)}\right\rangle+\left\langle\Delta I_{3}^{(i)} \Delta I_{3}^{(o)}\right\rangle=2 k_{\mathrm{B}} T G(\varepsilon)$

with

$G(\varepsilon)=\frac{2 e^{2}}{h}\left[\mathcal{T}_{1} \mathcal{T}_{2}+\mathcal{R}_{1} \mathcal{R}_{2}+2 \gamma(1-\varepsilon) \sqrt{\mathcal{T}_{1} \mathcal{T}_{2} \mathcal{R}_{1} \mathcal{R}_{2}} \cos \left(\Delta \phi_{A B}\right)\right]$

where

$\gamma=\pi k_{\mathrm{B}} T \Delta L /\left\{\hbar v_{D} \sinh \left[\pi k_{\mathrm{B}} T \Delta L /\left(\hbar v_{D}\right)\right]\right\}$

This is thus recognized as being the Johnson-Nyquist (equilibrium) contribution to the noise. Furthermore, we obtain

$\left\langle\Delta I_{3}^{(o)} \Delta I_{3}^{(o)}\right\rangle=\frac{2 e^{2}}{h} \int d E\{\alpha \cos (\phi)+\beta \cos (2 \phi)+\gamma+\delta\}$

with

$$
\begin{aligned}
\alpha= & (1-\varepsilon) \sqrt{\mathcal{R}_{1} \mathcal{R}_{2} \mathcal{T}_{1} \mathcal{T}_{2}}\left(f_{1}-f_{2}\right)\left[2\left(f_{1}-f_{2}\right)\left(\mathcal{R}_{1} \mathcal{R}_{2}+\mathcal{T}_{1} \mathcal{T}_{2}\right)+2 f_{2}-1\right] \\
\beta= & 2(1-\varepsilon)^{2} \mathcal{R}_{1} \mathcal{R}_{2} \mathcal{T}_{1} \mathcal{T}_{2}\left(f_{1}-f_{2}\right)^{2} \\
\gamma= & 2(1-\varepsilon)^{2} \mathcal{R}_{1} \mathcal{R}_{2} \mathcal{T}_{1} \mathcal{T}_{2}\left(f_{1}-f_{2}\right)^{2} \\
& +(1-X)\left[f_{1}\left(1-2 X f_{2}\right)-f_{1}^{2}(1-X)\right]+f_{2} X\left(1-X f_{2}\right) \\
& -\varepsilon \mathcal{R}_{2} \mathcal{T}_{2}\left[f_{1}+2(\varepsilon-2) f_{1} f_{2}+f_{2}\right] \\
\delta= & \varepsilon \mathcal{R}_{2} \mathcal{T}_{2}\left[f_{p_{o \uparrow}}\left(1-\varepsilon f_{p_{o \downarrow}}\right)+f_{p_{o \downarrow}}\left(1-\varepsilon f_{p_{o \uparrow}}\right)\right] \\
& +2 \varepsilon(1-\varepsilon) \mathcal{R}_{2} \mathcal{T}_{2}\left\{\left[f_{1} \mathcal{R}_{1}-f_{2} \mathcal{T}_{1}\right] f_{p_{o \uparrow}}-\left[f_{2} \mathcal{R}_{1}-f_{1} \mathcal{T}_{1}\right] f_{p_{o \downarrow}}\right\}
\end{aligned}
$$

where $X=\mathcal{R}_{1} \mathcal{T}_{2}+\mathcal{R}_{2} \mathcal{T}_{1}$. The zero frequency noise of the outer ES thus depends explicitly on the distribution functions of the probes via $\delta$. Let us look at some limits. In the fully coherent limit $\varepsilon=0$ we obtain

$$
\begin{aligned}
S_{33}= & \frac{2 e^{2}}{h} \int d E\left\{X^{2} f_{2}\left(1-f_{2}\right)+(1-X)^{2} f_{1}\left(1-f_{1}\right)+X(1-X)\left[f_{1}\left(1-f_{2}\right)+f_{2}\left(1-f_{1}\right)\right]\right. \\
& +2 \sqrt{\mathcal{T}_{1} \mathcal{T}_{2} \mathcal{R}_{1} \mathcal{R}_{2}}\left[f_{2}\left(1-f_{1}\right)+f_{1}\left(1-f_{2}\right)-2 f_{1}\left(1-f_{1}\right)-2 X\left(f_{1}-f_{2}\right)^{2}\right] \cos (\phi) 4 \mathcal{T}_{1} \mathcal{T}_{2} \mathcal{R}_{1} \\
& \left.\mathcal{R}_{2}\left(f_{1}-f_{2}\right)^{2} \cos ^{2}(\phi)\right\} .
\end{aligned}
$$

Thus the noise of the coherent interferometer displays first and second harmonic oscillations as a function of flux. At zero temperature and neglecting the energy dependence of the phase, this expression reduces to $S_{33}=\left(e^{2} \Delta \mu / h\right)[\langle T\rangle(1-\langle\mathcal{T}\rangle)]$ with $\langle\mathcal{T}\rangle=\mathcal{T}_{1} \mathcal{T}_{2}+\mathcal{R}_{1} \mathcal{R}_{2}+2 \sqrt{\mathcal{R}_{1} \mathcal{R}_{2} \mathcal{T}_{1} \mathcal{T}_{2}} \cos (\phi)$, consistent with [34].

For complete relaxation $\varepsilon=1$ and $\mathcal{T}_{1}=\mathcal{T}_{2} \equiv \mathcal{T}$, we find

$$
\left\langle\Delta I_{3}^{(o)} \Delta I_{3}^{(o)}\right\rangle=\left(2 e^{2} / h\right) \mathcal{T}(1-\mathcal{T}) \int d E\left[f_{p_{0 \uparrow}}\left(1-f_{p_{0 \downarrow}}\right)+f_{p_{0 \downarrow}}\left(1-f_{p_{0 \uparrow}}\right)\right]+F\left(f_{1}, f_{2}\right)
$$

with

$$
\begin{aligned}
& F\left(f_{1}, f_{2}\right)=\frac{2 e^{2}}{h} \int d E\left\{\left(\mathcal{R}^{2}+\mathcal{T}^{2}\right)^{2} f_{1}\left(1-f_{1}\right)+4(\mathcal{R} \mathcal{T})^{2} f_{2}\left(1-f_{2}\right)\right. \\
& \left.\quad+\mathcal{R T}(1-4 \mathcal{R})\left[f_{1}\left(1-f_{2}\right)+f_{2}\left(1-f_{1}\right)\right]\right\} \\
& \quad=\frac{2 e^{2}}{h}\left[\left\{\left(\mathcal{R}^{2}+\mathcal{T}^{2}\right)^{2}+4(\mathcal{R T})^{2}\right\} k \mathrm{~B} T+\mathcal{R T}(1-4 \mathcal{R} \mathcal{T}) \Delta \mu \operatorname{coth}\left(\frac{\Delta \mu}{2 k_{\mathrm{B}} T}\right)\right] .
\end{aligned}
$$

The probes all have the same temperature, given by Eq. (24), 
Integrating we find

$\int d E f_{p_{o \uparrow}}\left(1-f_{p_{o \downarrow}}\right)=\Delta \mu(1-2 \mathcal{T}) n_{B}\left(\frac{\Delta \mu(1-2 \mathcal{T})}{k_{\mathrm{B}} T_{p}}\right)$,

where $n_{B}(x)=1 /[\exp (x)-1]$ is the Bose function. Here we have used that $\mu_{p_{o \uparrow}}-\mu_{p_{o \downarrow}}=\Delta \mu(1-2 \mathcal{T})$, with $\Delta \mu=\mu_{2}-\mu_{1}$. Therefore we find

$$
\begin{aligned}
\left\langle\Delta I_{3}^{(o)} \Delta I_{3}^{(o)}\right\rangle= & \frac{2 e^{2}}{h} \mathcal{T}(1-\mathcal{T}) \Delta \mu(1-2 \mathcal{T}) \operatorname{coth}\left(\frac{\Delta \mu(1-2 \mathcal{T})}{2 k_{\mathrm{B}} T_{p}}\right) \\
& +F\left(f_{1}, f_{2}\right) .
\end{aligned}
$$

Thus energy conservation of the relaxation process modifies current correlations in the Mach-Zehnder interferometer.

In the zero temperature limit we have $k_{\mathrm{B}} T_{p}=|\Delta \mu| \sqrt{3 \mathcal{R T} /\left(2 \pi^{2}\right)}$ and so the full current noise in contact 3 at zero temperature is

$$
\begin{aligned}
& \left.\left\langle\Delta I_{3} \Delta I_{3}\right\rangle\right|_{k_{\mathrm{B}} T=0}=\frac{2 e^{2}}{h} \mid \Delta \mu \\
& \mathbb{R} \mathcal{T}\left\{(\mathcal{R}-\mathcal{T}) \operatorname{coth}\left(\frac{\pi}{\sqrt{6}} \frac{\mathcal{R}-\mathcal{T}}{\sqrt{\mathcal{R T}}}\right)+1-4 \mathcal{R T}\right\} .
\end{aligned}
$$

Expression (37) is represented graphically as a function of $\mathcal{T}$ in Fig. 6 (red curve), where we compare it with the result $S_{33}=2 \mathcal{R} \mathcal{T}\left[\mathcal{R}^{2}+\mathcal{T}^{2}-\min (\mathcal{R}, \mathcal{T})\right]$, obtained [34] for uncorrelated, equally coupled probes with temperatures $T_{p}=T$ (dashed blue curve) and with a classical non-interacting exclusion model $[13,34]$ where the noise $S_{33}=2 \mathcal{R T}\left(\mathcal{R}^{2}+\mathcal{T}^{2}\right)-2(\mathcal{R T})^{2}$ is derived [34] by summing scattering probabilities and taking into account the Pauli principle ${ }^{4}$ (dotted black curve). Incidentally, the latter expression is identical to the one obtained from a dephasing probe model, which conserves energy in each ES, in the fully dephased limit $[13,34]$. We see that compared to the uncorrelated voltage probes, the noise here is larger and never suppressed to zero (apart for $\mathcal{R}=0,1$ ) although there is a local minimum for $\mathcal{R}=\mathcal{T}$. Compared with the exclusion model, the noise in the CVP model is suppressed.

\section{Conclusion}

In this work we have introduced a phenomenological model to describe energy relaxation of two interacting mesoscopic systems. The model, which extends the voltage probe model [1-3], is built such as to conserve the total energy in the system. We use this model to compute the distribution functions of two Coulomb coupled ESs in the integer quantum Hall regime and to investigate current noise through a chiral Mach-Zehnder interferometer with two co-propagating interacting channels. Although this model cannot explain the origin of the relaxation mechanism, its predictions may easily be tested experimentally.

While the zero frequency noise of non-interfering ESs is insensitive to energy relaxation as mediated by the correlated voltage probes, we find that the current noise through a MachZehnder interferometer is sensitive to the energy distribution in the probes via a term which is proportional to the noise of the second QPC. In particular this term is sensitive to the effective temperature of the probes determined by the requirement of energy conservation of the relaxation process.

\footnotetext{
${ }^{4}$ As argued in [13], for an interferometer with different arm lengths, such that $\Delta L>h v_{D} /(\mathrm{eV})$, the probability that two particles arrive simultaneously at the second QPC is not negligible and one should take the Pauli principle into account. As a consequence the classical noise is suppressed [13,34] by a term $-2 \mathcal{R}_{1} \mathcal{T}_{1} \mathcal{R}_{2} \mathcal{T}_{2}$.
}

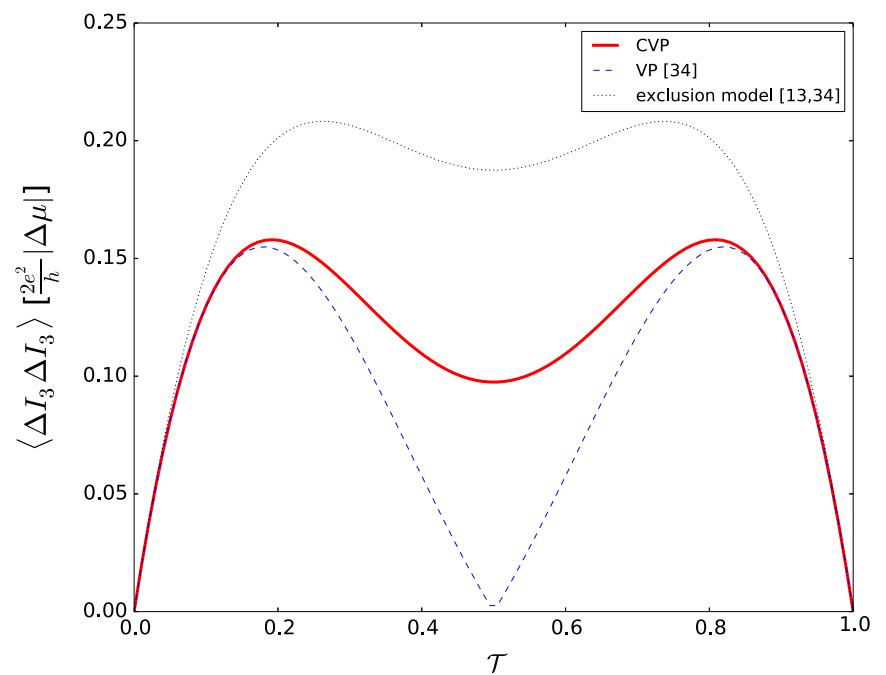

Fig. 6. Full (red) curve: $\left\langle\Delta I_{3} \Delta I_{3}\right\rangle$ according to formula (37) as a function of $\mathcal{T}=\mathcal{T}_{1}=\mathcal{T}_{2}$ for $\varepsilon=1$ and $k_{\mathrm{B}} T=0$. Dashed (blue) curve: the corresponding noise into probe 3 for $T_{p}=T$. $\Delta \mu=1 \mathrm{a}$. u. Dotted (black) curve: the corresponding noise of a classical non-interacting Mach-Zehnder setup. (For interpretation of the references to color in this figure caption, the reader is referred to the web version of this paper.)

\section{Acknowledgments}

This work was financially supported by the Swiss National Science Foundation Ambizione Grant no. PZ00P2_148092.

\section{Appendix A. Some Fermi function integrals}

(a) We show that $\int d E\left[f_{a}(E-\mu)-f_{b}(E)\right]=\mu \quad$ where $f_{\alpha}(E)=1 /[\exp (\alpha E)+1]$. We have

$$
\begin{aligned}
& \int d E\left[f_{a}(E-\mu)-f_{b}(E)\right] \\
& =\lim _{R \rightarrow \infty}\left\{\frac{1}{b} \ln \left[\frac{1+e^{b R}}{1+e^{-b R}}\right]-\frac{1}{a} \ln \left[\frac{1+e^{a(R-\mu)}}{1+e^{-a(R+\mu)}}\right]\right\} \\
& =\lim _{R \rightarrow \infty} \ln \left\{\left(\frac{1+e^{b R}}{1+e^{-b R}}\right)^{1 / b}\left(\frac{1+e^{-a(R+\mu)}}{1+e^{a(R-\mu)}}\right)^{1 / a}\right\} \\
& =\lim _{R \rightarrow \infty} \ln \left\{\left(e^{b R}\right)^{1 / b}\left(\frac{1}{e^{a(R-\mu)}}\right)^{1 / a}\right\}=\lim _{R \rightarrow \infty} \ln \left\{\frac{e^{R}}{e^{(R-\mu)}}\right\}=\mu .
\end{aligned}
$$

(b) We next evaluate the integral $J=\int d E E\left[f_{a}(E-\mu)-f_{b}(E)\right]$. We have

$$
\begin{aligned}
J= & \lim _{R \rightarrow \infty}\left\{\frac{R}{b} \ln \left[\left(1+e^{b R}\right)\left(1+e^{-b R}\right)\right]\right. \\
& -\frac{R}{a} \ln \left[\left(1+e^{a(R-\mu)}\right)\left(1+e^{-a(R+\mu)}\right)\right] \\
& +\frac{1}{b^{2}}\left[\operatorname{Li}_{2}\left(-e^{b R}\right)-\operatorname{Li}_{2}\left(-e^{-b R}\right)\right] \\
& \left.-\frac{1}{a^{2}}\left[\operatorname{Li}_{2}\left(-e^{a(R-\mu)}\right)-\operatorname{Li}_{2}\left(-e^{-a(R+\mu)}\right)\right]\right\},
\end{aligned}
$$

with the dilogarithm $\operatorname{Li}_{2}(z)=\sum_{n=1}^{\infty} z^{n} / n^{2}$. The first two terms can be evaluated straightforwardly as above and since $\mathrm{Li}_{2}(0)=0$ and using the reflection property [51] $\mathrm{Li}_{2}(z)=-\mathrm{Li}_{2}(1 / z)-\pi^{2} / 6-(1 / 2) \ln ^{2}(-1 / z)$, we obtain 


$$
\begin{aligned}
J= & \lim _{R \rightarrow \infty}\left\{\mu R+\frac{1}{b^{2}} \operatorname{Li}_{2}\left(-e^{b R}\right)-\frac{1}{a^{2}} \operatorname{Li}_{2}\left(-e^{a[R-\mu]}\right)\right\} \\
= & \lim _{R \rightarrow \infty}\left\{\mu R-\frac{\pi^{2}}{6 b^{2}}-\frac{1}{2 b^{2}} \ln ^{2}\left(e^{-b R}\right)+\frac{\pi^{2}}{6 a^{2}}\right. \\
& \left.+\frac{1}{2 a^{2}} \ln ^{2}\left(e^{-a[R-\mu]}\right)\right\} \\
= & \lim _{R \rightarrow \infty}\left\{\mu R+\frac{\pi^{2}}{6}\left(\frac{1}{a^{2}}-\frac{1}{b^{2}}\right)-\frac{R^{2}}{2}+\frac{(R-\mu)^{2}}{2}\right\} \\
= & \frac{\mu^{2}}{2}+\frac{\pi^{2}}{6}\left(\frac{1}{a^{2}}-\frac{1}{b^{2}}\right) .
\end{aligned}
$$

(c) Here we evaluate the integral $H=\int d E \cos (a+b E) f_{\beta}{ }^{\prime}(E)$, with the Fermi function derivative $f_{\beta}^{\prime}(E)=-\beta e^{\beta E} /\left(e^{\beta E}+1\right)^{2}=$ $-\beta f_{\beta}(E)\left(1-f_{\beta}(E)\right)$. Making the substitution $z=e^{\beta E}$ and introducing $\eta=b / \beta$, we have

$$
H=-\int_{0}^{\infty} d z \frac{\cos (a+\eta \ln z)}{(z+1)^{2}} \text {. }
$$

Next we use Euler's formula to rewrite this integral as

$$
\begin{aligned}
H= & -\frac{e^{i a}}{2} \int_{0}^{\infty} d z \frac{\exp [i \eta \ln (z)]}{(z+1)^{2}} \\
& -\frac{e^{-i a}}{2} \int_{0}^{\infty} d z \frac{\exp [-i \eta \ln (z)]}{(z+1)^{2}} \\
H= & -\frac{e^{i a}}{2} \int_{0}^{\infty} d z \frac{z^{i \eta}}{(z+1)^{2}}-\frac{e^{-i a}}{2} \int_{0}^{\infty} d z \frac{z^{-i \eta}}{(z+1)^{2}} .
\end{aligned}
$$$$
\text { Since } \quad \int_{0}^{\infty} d z \frac{z^{i \eta}}{(z+1)^{2}}=\int_{0}^{\infty} d z \frac{z^{-i \eta}}{(z+1)^{2}}=\pi \eta \operatorname{csch}(\pi \eta) \text {, }
$$

$\operatorname{csch}(x)=1 / \sinh (x)$, we finally obtain

$H=-\pi \eta \frac{\cos (a)}{\sinh (\pi \eta)}$.

To second order in $\eta$ this yields

$$
H \approx \cos (a)\left(\frac{\pi^{2}}{6} \eta^{2}-1\right) \text {. }
$$

\section{References}

[1] M. Büttiker, Small normal-metal loop coupled to an electron reservoir, Phys. Rev. B 32 (1985) 1846-1849, http://dx.doi.org/10.1103/PhysRevB.32.1846 (URL 〈http://link.aps.org/doi/10.1103/PhysRevB.32.1846〉).

[2] M. Büttiker, Role of quantum coherence in series resistors, Phys. Rev. B 33 (1986) 3020.

[3] M. Büttiker, Coherent and sequential tunneling in series barriers, IBM J. Res. Dev. 32 (1988) 63.

[4] H.-P. Breuer, F. Petruccione, The Theory of Open Quantum Systems, Oxford University Press, New York, 2007.

[5] P.W. Brouwer, C.W.J. Beenakker, Voltage-probe and imaginary-potential models for dephasing in a chaotic quantum dot, Phys. Rev. B 55 (1997) 4695-4702, http://dx.doi.org/10.1103/PhysRevB.55.4695 (URL 〈http://link.aps. org/doi/10.1103/PhysRevB.55.4695〉).

[6] H.U. Baranger, A.D. Stone, D.P. DiVincenzo, Resistance fluctuations in multiprobe microstructures: length dependence and nonlocality, Phys. Rev. B 37 (1988) 6521-6524, http://dx.doi.org/10.1103/PhysRevB.37.6521 (URL 〈http:// link.aps.org/doi/10.1103/PhysRevB.37.6521>)

[7] C.L. Kane, P.A. Lee, D.P. DiVincenzo, Voltage fluctuations in multilead devices, Phys. Rev. B 38 (1988) 2995-3005, http://dx.doi.org/10.1103/PhysRevB.38.2995 (URL 〈http://link.aps.org/doi/10.1103/PhysRevB.38.2995〉).

[8] D. Kowal, U. Sivan, O. Entin-Wohlman, Y. Imry, Transmission through multiplyconnected wire systems, Phys. Rev. B 42 (1990) 9009-9018, http://dx.doi.org/ 10.1103/PhysRevB.42.9009 (URL 〈http://link.aps.org/doi/10.1103/PhysRevB.42. 9009)).

[9] S. Hershfield, Equivalence of the multilead approach to dephasing and the self-consistent born approximation, Phys. Rev. B 43 (1991) 11586-11594, http: //dx.doi.org/10.1103/PhysRevB.43.11586 (URL 〈http://link.aps.org/doi/10.1103/ PhysRevB.43.11586>).

[10] P.A. Mello, Scattering approach to persistent currents in infinitely extended quantum systems, Phys. Rev. B 47 (1993) 16358-16368, http://dx.doi.org/ 10.1103/PhysRevB.47.16358 (URL 〈http://link.aps.org/doi/10.1103/PhysRevB.47.
16358)).

11] P.W. Brouwer, C.W.J. Beenakker, Effect of a voltage probe on the phase-coherent conductance of a ballistic chaotic cavity, Phys. Rev. B 51 (1995) 7739-7743, http://dx.doi.org/10.1103/PhysRevB.51.7739 (URL 〈http://link.aps. org/doi/10.1103/PhysRevB.51.7739)).

[12] B. Michaelis, C.W.J. Beenakker, Phys. Rev. B 73 (2006) 115329.

[13] S. Pilgram, P. Samuelsson, H. Förster, M. Büttiker, Full counting statistics for voltage and dephasing probes, Phys. Rev. Lett. 97 (2006) 066801.

[14] H. Förster, P. Samuelsson, S. Pilgram, M. Büttiker, Voltage and dephasing probes: a full counting statistics discussion, Phys. Rev. B 75 (2007) 035340.

[15] S.E. Nigg, M. Büttiker, Quantum to classical transition of the charge relaxation resistance of a mesoscopic capacitor, Phys. Rev. B 77 (2008) 085312, http://dx. doi.org/10.1103/PhysRevB.77.085312 (URL 〈http://link.aps.org/doi/10.1103/ PhysRevB.77.085312〉).

[16] C.M. Marcus, R.M. Westervelt, P.F. Hopkins, A.C. Gossard, Phase breaking in ballistic quantum dots: experiment and analysis based on chaotic scattering Phys. Rev. B 48 (1993) 2460-2464, http://dx.doi.org/10.1103/PhysRevB.48.2460 (URL 〈http://link.aps.org/doi/10.1103/PhysRevB.48.2460〉).

[17] P. Roulleau, F. Portier, P. Roche, A. Cavanna, G. Faini, U. Gennser, D. Mailly, Tuning decoherence with a voltage probe, Phys. Rev. Lett. 102 (2009) 236802 http://dx.doi.org/10.1103/PhysRevLett.102.236802 (URL 〈http://link.aps.org/ doi/10.1103/PhysRevLett.102.236802)).

[18] P.M. Brouwer, A. Lamacraft, K. Flensberg, Phys. Rev. B 72 (2005) 075316.

[19] S.E. Nigg, R. López, M. Büttiker, Mesoscopic charge relaxation, Phys. Rev. Lett. 97 (2006) 206804, http://dx.doi.org/10.1103/PhysRevLett.97.206804.

[20] A.V. Lebedev, G.B. Lesovik, G. Blatter, $n$-Particle scattering matrix for electrons interacting on a quantum dot, Phys. Rev. Lett. 100 (2008) 226805, http://dx. doi.org/10.1103/PhysRevLett.100.226805 (URL <http://link.aps.org/doi/10.1103/ PhysRevLett.100.226805〉).

[21] D. Oehri, A.V. Lebedev, G.B. Lesovik, G. Blatter, Scattering matrix approach to interacting electron transport, Phys. Rev. B 86 (2012) 125301, http://dx.doi. org/10.1103/PhysRevB.86.125301 (URL 〈http://link.aps.org/doi/10.1103/Phys RevB.86.125301〉).

[22] P. Degiovanni, C. Grenier, G. Fève, Decoherence and relaxation of singleelectron excitations in quantum Hall edge channels, Phys. Rev. B 80 (2009) 241307, http://dx.doi.org/10.1103/PhysRevB.80.241307 (URL 〈http://link.aps. org/doi/10.1103/PhysRevB.80.241307〉).

[23] H. le Sueur, C. Altimiras, U. Gennser, A. Cavanna, D. Mailly, F. Pierre, Energy relaxation in the integer quantum Hall regime, Phys. Rev. Lett. 105 (5) (2010) 056803, http://dx.doi.org/10.1103/PhysRevLett.105.056803.

[24] C. Altimiras, H. le Sueur, U. Gennser, A. Cavanna, D. Mailly, F. Pierre, Tuning energy relaxation along quantum Hall channels, Phys. Rev. Lett. 105 (22) (2010) 226804, http://dx.doi.org/10.1103/PhysRevLett.105.226804.

[25] A.M. Lunde, S.E. Nigg, M. Büttiker, Interaction-induced edge channel equilibration, Phys. Rev. B 81 (2010) 041311, http://dx.doi.org/10.1103/PhysRevB.81.041311 (URL 〈http://link.aps.org/doi/10.1103/PhysRevB.81.041311〉).

[26] P. Degiovanni, C. Grenier, G. Fève, C. Altimiras, H. le Sueur, F. Pierre, Plasmon scattering approach to energy exchange and high-frequency noise in $\nu=2$ quantum Hall edge channels, Phys. Rev. B 81 (2010) 121302, http://dx.doi.org/ 10.1103/PhysRevB.81.121302 (URL 〈http://link.aps.org/doi/10.1103/PhysRevB. 81.121302)).

[27] I.P. Levkivskyi, E.V. Sukhorukov, Energy relaxation at quantum Hall edge, Phys Rev. B 85 (2012) 075309, http://dx.doi.org/10.1103/PhysRevB.85.075309 (URL 〈http://link.aps.org/doi/10.1103/PhysRevB.85.075309)).

[28] T. Karzig, A. Levchenko, L.I. Glazman, F. von Oppen, Relaxation and edge reconstruction in integer quantum Hall systems, N. J. Phys. 14 (10) (2012) 105009 (URL 〈http://stacks.iop.org/1367-2630/14/i=10/a =105009/).

[29] E. Bocquillon, V. Freulon, F.D. Parmentier, J.-M. Berroir, B. Plaais, C. Wahl, J. Rech, T. Jonckheere, T. Martin, C. Grenier, D. Ferraro, P. Degiovanni, G. Fve, Electron quantum optics in ballistic chiral conductors, Ann. Phys. 526 (1-2) (2014) 1-30, http://dx.doi.org/10.1002/andp.201300181.

[30] T. Otsuka, Y. Sugihara, J. Yoneda, T. Nakajima, S. Tarucha, Measurement of energy relaxation in quantum Hall edge states utilizing quantum point contacts, J. Phys. Soc. Jpn. 83 (1) (2014) 014710, http://dx.doi.org/10.7566/ JPSJ.83.014710.

[31] H. Inoue, A. Grivnin, N. Ofek, I. Neder, M. Heiblum, V. Umansky, D. Mahalu, Charge fractionalization in the integer quantum Hall effect, Phys. Rev. Lett. 112 (2014) 166801, http://dx.doi.org/10.1103/PhysRevLett.112.166801 (URL 〈http:// link.aps.org/doi/10.1103/PhysRevLett.112.166801>).

[32] D. Ferraro, B. Roussel, C. Cabart, E. Thibierge, G. Fève, C. Grenier, P. Degiovanni, Real-time decoherence of Landau and Levitov quasiparticles in quantum Hall edge channels, Phys. Rev. Lett. 113 (2014) 166403, http://dx.doi.org/10.1103/ PhysRevLett.113.166403 (URL 〈http://link.aps.org/doi/10.1103/PhysRevLett.113. 166403)).

[33] C. Texier, M. Büttiker, Effect of incoherent scattering on shot noise correlations in the quantum Hall regime, Phys. Rev. B 62 (2000) 7454-7458, http://dx.doi. org/10.1103/PhysRevB.62.7454 (URL 〈http://link.aps.org/doi/10.1103/PhysRevB. $62.7454\rangle)$.

[34] F. Marquardt, C. Bruder, Influence of dephasing on shot noise in an electronic Mach-Zehnder interferometer, Phys. Rev. Lett. 92 (2004) 056805, http://dx. doi.org/10.1103/PhysRevLett.92.056805 (URL 〈http://link.aps.org/doi/10.1103/ PhysRevLett.92.056805〉).

[35] I. Neder, M. Heiblum, Y. Levinson, D. Mahalu, V. Umansky, Unexpected behavior in a two-path electron interferometer, Phys. Rev. Lett. 96 (2006) 016804, http://dx.doi.org/10.1103/PhysRevLett.96.016804 (URL 〈http://link. aps.org/doi/10.1103/PhysRevLett.96.016804〉). 
[36] L.V. Litvin, H.-P. Tranitz, W. Wegscheider, C. Strunk, Decoherence and single electron charging in an electronic Mach-Zehnder interferometer, Phys. Rev. B 75 (2007) 033315, http://dx.doi.org/10.1103/PhysRevB.75.033315 (URL 〈http:// link.aps.org/doi/10.1103/PhysRevB.75.033315>).

[37] P. Roulleau, F. Portier, D.C. Glattli, P. Roche, A. Cavanna, G. Faini, U. Gennser, D. Mailly, Finite bias visibility of the electronic Mach-Zehnder interferometer, Phys. Rev. B 76 (2007) 161309, http://dx.doi.org/10.1103/PhysRevB.76.161309 (URL 〈http://link.aps.org/doi/10.1103/PhysRevB.76.161309)).

[38] J.T. Chalker, Y. Gefen, M.Y. Veillette, Decoherence and interactions in an electronic Mach-Zehnder interferometer, Phys. Rev. B 76 (2007) 085320, http: //dx.doi.org/10.1103/PhysRevB.76.085320 (URL 〈http://link.aps.org/doi/10. 1103/PhysRevB.76.085320)).

[39] E.V. Sukhorukov, V.V. Cheianov, Resonant dephasing in the electronic MachZehnder interferometer, Phys. Rev. Lett. 99 (2007) 156801, http://dx.doi.org/ 10.1103/PhysRevLett.99.156801 (URL 〈http://link.aps.org/doi/10.1103/PhysRev Lett.99.156801〉).

[40] C. Neuenhahn, F. Marquardt, Dephasing by electron-electron interactions in a ballistic Mach-Zehnder interferometer, N. J. Phys. 10 (11) (2008) 115018 (URL 〈http://stacks.iop.org/1367-2630/10/i=11/a=115018〉).

[41] I.P. Levkivskyi, E.V. Sukhorukov, Dephasing in the electronic Mach-Zehnder interferometer at filling factor $\nu=2$, Phys. Rev. B 78 (2008) 045322, http://dx doi.org/10.1103/PhysRevB.78.045322 (URL 〈http://link.aps.org/doi/10.1103/ PhysRevB.78.045322>).

[42] I. Neder, E. Ginossar, Behavior of electronic interferometers in the nonlinear regime, Phys. Rev. Lett. 100 (2008) 196806, http://dx.doi.org/10.1103/PhysRevLett.100.196806 (URL 〈http://link.aps.org/doi/10.1103/PhysRevLett.100.
196806)).

[43] P. Roulleau, F. Portier, P. Roche, A. Cavanna, G. Faini, U. Gennser, D. Mailly, Direct measurement of the coherence length of edge states in the integer quantum Hall regime, Phys. Rev. Lett. 100 (2008) 126802, http://dx.doi.org/ 10.1103/PhysRevLett.100.126802 (URL 〈http://link.aps.org/doi/10.1103/Phys RevLett.100.126802)).

[44] H.C. Lee, Influence of long range coulomb interaction on the electronic MachZehnder interferometer of quantum Hall edge states, Phys. Rev. B 79 (2009) 155435, http://dx.doi.org/10.1103/PhysRevB.79.155435 (URL 〈http://link.aps. org/doi/10.1103/PhysRevB.79.155435)).

[45] D.L. Kovrizhin, J.T. Chalker, Equilibration of integer quantum Hall edge states Phys. Rev. B 84, 085105 http://dx.doi.org/10.1103/PhysRevB.84.085105.

[46] D.L. Kovrizhin, J.T. Chalker, Relxation in Driven Integer Quantum Hall Edge States (URL 〈http://arxiv.org/pdf/1111.3914)).

[47] T. Christen, M. Büttiker, Low-frequency admittance of quantized Hall conductors, Phys. Rev. B 53 (1996) 2064-2072, http://dx.doi.org/10.1103/PhysRevB.53.2064 (URL 〈http://link.aps.org/doi/10.1103/PhysRevB.53.2064〉).

[48] Y.M. Blanter, M. Büttiker, Phys. Rep. 336 (2000).

[49] S.-W.V. Chung, M. Moskalets, P. Samuelsson, Phys. Rev. B 75 (2007) 115332.

[50] M. Hashisaka, H. Kamata, N. Kumada, K. Washio, R. Murata, K. Muraki, T. Fujisawa, Distributed-element circuit model of edge magnetoplasmon transport, Phys. Rev. B 88 (2013) 235409, http://dx.doi.org/10.1103/PhysRevB.88.235409 (URL 〈http://link.aps.org/doi/10.1103/PhysRevB.88.235409)).

[51] D. Sagier, Frontiers in Number Theory, Physics and Geometry II, SpringerVerlag, Berlin, Heidelberg, New York, 2006. 